\title{
Ubiquitous Switching in Molecular Wires
}

\author{
G. K. Ramachandran ${ }^{*}$, Theresa Hopson ${ }^{* *}$, Adam Rawlett ${ }^{* *}$, Larry Nagahara ${ }^{* *}$, Stuart M Lindsay ${ }^{*}$ \\ * Department of Physics \& Astronomy, Arizona State University, Tempe, AZ 85287 \\ ** Physical Sciences Research Labs, Motorola Labs, 7700 S River Pkwy, Tempe, AZ 85284
}

Stochastic on-off switching has been reported [1,2] in STM (scanning tunneling microscopy) experiments in phenylene-ethynylene oligomers. Switching is characterized by two states, "on" and "off", based on apparent height differences in temporally sequential topographic images collected with the STM. The mechanism(s) inducing on-off behavior is unclear yet, however, either conformational changes or mechanical changes in the film (or a combination of both) are speculated as possible mechanisms [1,2]. In STM experiments height differences cannot be unambiguously attributed to either a conductivity change or a mechanical height change in the system under study (Scheme 1).

In our efforts to understand the underlying mechanism(s) responsible for stochastic switching in wired molecules, we serendipitously discovered that even the simplest wired molecules, $n$-alkane dithiols [inserted, and isolated in $n$-alkane monothiol monolayers and bonded on $\mathrm{Au}$ (111)] exhibit switching. We show using STM and CAFM (current sensing atomic force microscopy) that the mechanism also responsible for molecular switching is due to a subtle phenomenon: the instability in the Au-S bond that binds the molecules to Au (111) surfaces. Additionally, we predict that any wired molecule will undergo stochastic switching.

Figure $1 \mathrm{~A}-\mathrm{C}$ shows reversible switching in sequential images of octane dithiol (C8d) inserted in an octane monothiol (C8m) self-assembled-monolayer (SAM). Reversible switching is also seen in decane dithiol (C10d in a C10m SAM) and in dodecane dithiol (C12d) inserted in a C12m SAM (Figure 1 D-F and G-I). Scheme 1 shows the working of an STM: the tip scans the surface without physical contact with the molecule to maintain a constant tunnel current. Hence, one cannot uniquely deduce whether the switching is caused due to a physical tilt of the molecule or due to a conductivity change (or both).

In a CAFM experiment however (Scheme 2), a metal ( $\mathrm{Au}$ ) coated tip in physical contact with the surface follows the topography while simultaneously recording the current associated with the area in study. Unlike the STM where the servo follows the tunnel current, the CAFM follows a contour of constant force. Thus, any physical tilt of the molecule will not result in a loss of contact. In Figure 2 we show switching in sequential CAFM images of 1-nitro-2,5-di-(phenylethynyl-4')-benzenenano Au inserted in $\mathrm{C} 12 \mathrm{~m}$ SAMs. Because a tilt alone cannot cause a change in measured current (Figure 2B), we conclude that at least a fraction of the total switching events is caused by a mechanism not associated with tilt fluctuations.

As is clear from the arguments (based on switching in both STM and CAFM) above, at least a certain fraction of the overall switching events are a result of a new instability and not due to "tilt fluctuations" alone. We propose a thermally activated labile bottom [Au (111)-S] bond contact to be responsible for this instability. Based on tabulated values for the strength of the Au-S bond, we can estimate a "break frequency" $f$, with $f \propto v_{0} \times \exp (-\Delta \mathrm{E} / k \mathrm{~T})$, where $v_{0}$ is the Au-S vibrational frequency ( $\mathrm{THz}$ range) and $\Delta \mathrm{E}$ (eV range) represents the $\mathrm{Au}-\mathrm{S}$ bond strength.

The motivation behind this model is the fact that one may successfully explain (i) on-off behavior in systems as disparate as the $n$-alkane dithiols and the phenylene-ethynylene oligomers (or any other 
complex molecule that switches) and (ii) the observed switching in both STM and CAFM experiments. Unfortunately, we find that depending on our choice of $\Delta \mathrm{E}$ (determined at $40 \mathrm{kCal} / \mathrm{mol}$ for alkane monothiol monolayers) rates for switching can be varied between a few seconds to several ten thousands of seconds (meaning switching never occurs). An unambiguous determination of the switching rate is compounded by the fact that accurate $\mathrm{Au}-\mathrm{S}$ bond strengths are not known even for individual dithiol molecules leave alone what they may be in different environments (for example in different SAMs or in different packing densities [2]). Rate determinations based on estimates of torsional-barriers (relative rotation of rings) in phenylene-ethynylene oligomers pose a similar challenge - switching rates can estimated to be a few pico seconds or millions of years depending on neighboring interactions.

We have quantitatively arrived at the same conclusion exploiting the symmetry of the Au-Smolecule-S-(Au) junction. First, we realize that the $\mathrm{Au}-\mathrm{S}$ bond could break either at the top or bottom contact to the molecule. To eliminate the role of the top contact we attached Au nanoparticles to the free thiol end of the molecule, following Cui et al. Quantitative analysis of the normalized switching rates for several molecules connected in different ways reveals that a certain fraction of the switching events are a direct result of the S-Au (bottom contact) breaking.

\section{References}

[1] Donhauser Z. J.; Mantooth B. A.; Kelly K. F.; Bumm L. A.; Monnell J. D.; Stapleton J. J.; Price D. W.; Rawlett A. M.; Allara D. L.; Tour J. M.; Weiss P. M. Science 2001, 292, 2303-2307.

[2] Donhauser Z. J.; Mantooth B. A.; Pearl T. P.; Kelly K. F.; Nanayakkara S. U.; Weiss P. S. Jpn. J. App. Phys. 2002, 41, 4871-4877.
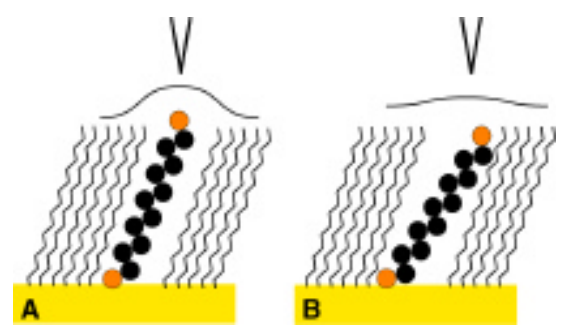

FIGURE 1. Switching in STM: octane dithiol, C8d (A-C), decane dithiol, C10d (D-F), and in dodecane dithiol, C12d (G-I).

SCHEME 1. In STM a metal tip (Pt-Ir) scans the surface, at a constant tunnel current (A). Any changes in the physical height of the molecule (B) may result in the observation of "on-off" switching.

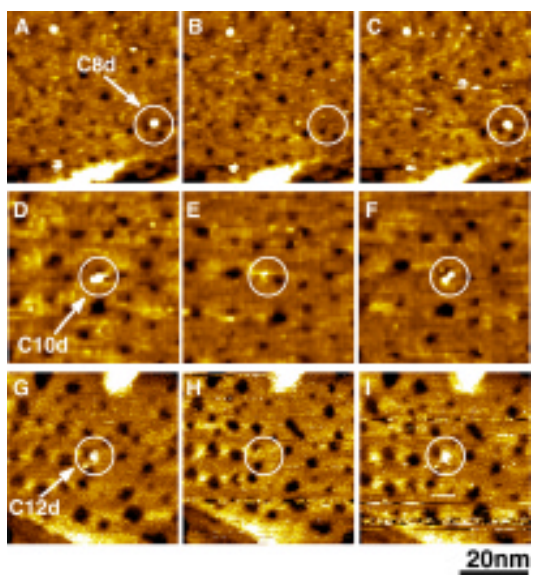

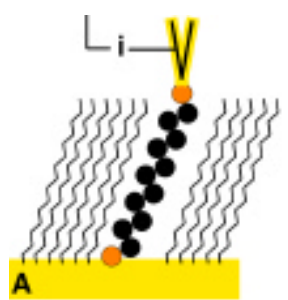

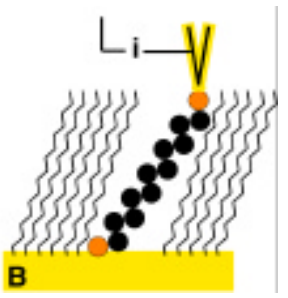

SCHEME 2. In CAFM, a metal coated tip (Au) scans the surface, at a constant force in contact with the surface (A). Any change in the physical height of the molecule (B) does not result in a loss of contact.
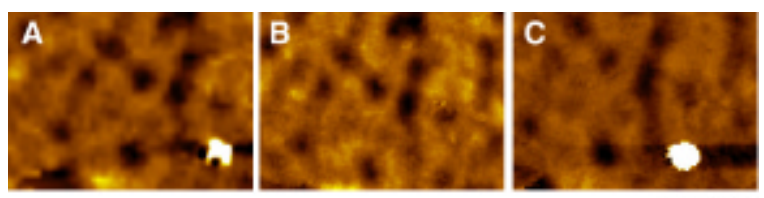

$30 \mathrm{~nm}$

FIGURE 2. Switching in CAFM experiments (shown are current images) in the 1-nitro-2,5-di-(phenylethynyl-4'thio)-benzene-nanoAu molecule. The molecule appears as bright spot ("on") in A, and disappears into the "off" state in $\mathrm{B}$, only to reappear in $\mathrm{C}$. 